\title{
MALIGNANT GASTROINTESTINAL STROMAL TUMOUR WITH
}

\section{METASTASIS}

Bhushita Lakhar ${ }^{1}$, Nilesh Guru

${ }^{1}$ Assistant Professor, Shri BM Patil Medical College, Hospital \& Research Centre, Bijapur, Karnataka, India

${ }^{2}$ Senior Resident, Department of Surgery, Shri BM Patil Medical College, Hospital \& Research Centre, Bijapur, Karnataka, India

Conflict of Interest - NIL, Received - 16/11/2015, Accepted - 19/11/2015, Published-20/11/2015

ABSTRACT: - Gastrointestinal stromal tumors (GISTs) are the most usual mesenchymal neoplasms of the gastrointestinal tract. Ever since the classification of GIST as an entity distinct from leiomyoma's, leiomyosarcomas, etc., there has been an increased concern in defining their imaging characteristics. It is estimated that approximately 5000-10,000 people are affected per year by this tumor all over the world. Most GISTs are benign (70-80\%). However, these tumors have a spectrum ranging from benign to malignant lesions, depending on its anatomic site, tumor size, and mitotic frequency. We report a case of multiple malignant GIST with metastasis into Liver.

KEYWORDS: - Hepatic metastasis, gastrointestinal stromal tumour, Liver

\section{INTRODUCTION}

Gastrointestinal stromal tumors (GISTs) are the most common mesenchymal neoplasms of the gastrointestinal tract. The term GIST defines a unique group of mesenchymal neoplasms that are distinct from true smooth muscle and neural tumors (1). Ever since the classification of GIST as an entity distinct from leiomyoma's, leiomyosarcomas, etc., there has been an increased interest in defining their imaging characteristics. It is estimated that approximately 5000-10,000 people are affected per year by this tumor all over the world. Most GISTs are benign (70-80\%).

However, these tumors have a spectrum ranging from benign to malignant lesions, depending on the anatomic site, tumor size, and mitotic frequency (1). We report a case of malignant GISTs with metastasis into Liver.

\section{CASE PRESENTATION}

A $45 \mathrm{yr}$ old male was referred to our department for CT Scan of his abdomen. He complained of abdominal pain and bleeding per rectum since 3 months. On examination he had diffuse abdominal pain and per rectal examination was normal. The laboratory tests were also normal.

On performing CT scan there were multiple illdefined hetrogenous exophytic masses with central necrosis abutting the jejunal loops and the caecum. The size of masses ranges from approx. $7 \mathrm{~cm}$ to 12 $\mathrm{cm}$. On contrast administration, these lesions showed heterogeneous peripheral enhancement (Figure 1 and 2). The caecum also showed significant wall thickening with narrowing of the lumen. The liver showed multiple well defined lobulated heterogeneously enhancing masses with central necrosis in left lobe, largest one measuring about $8.5 \times 6.5 \mathrm{~cm}$ (figure $1 \mathrm{~A}$ ). The lesion was extending outside the liver in anterior perihepatic 
space and gastro hepatic regions. These lesions were causing compression of left portal vein. There was minimal ascites present in the abdomen.

A possibility of malignant GIST with liver metastasis was kept. On histopathological correlation, this above diagnosis was confirmed to be malignant GISTs with liver metastasis.

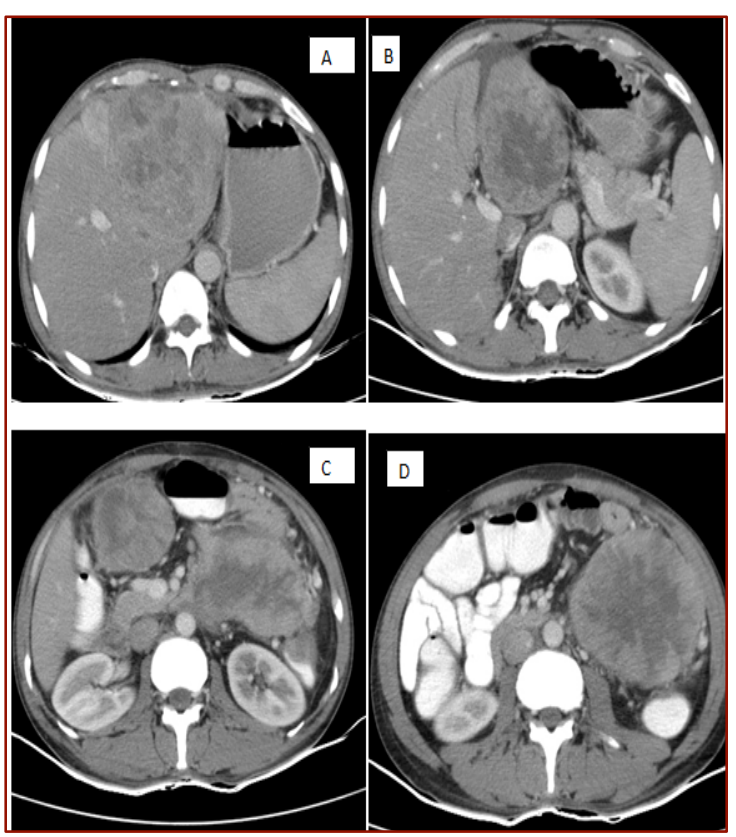

Figure Legend 1: A: Large heterogeneously enhancing mass lesion within left lobe of liver metastasis

Figure 1: B, C, and D: Large exophytic heterogeneously enhancing lesion in the peritoneal cavity abutting the small bowel loops.
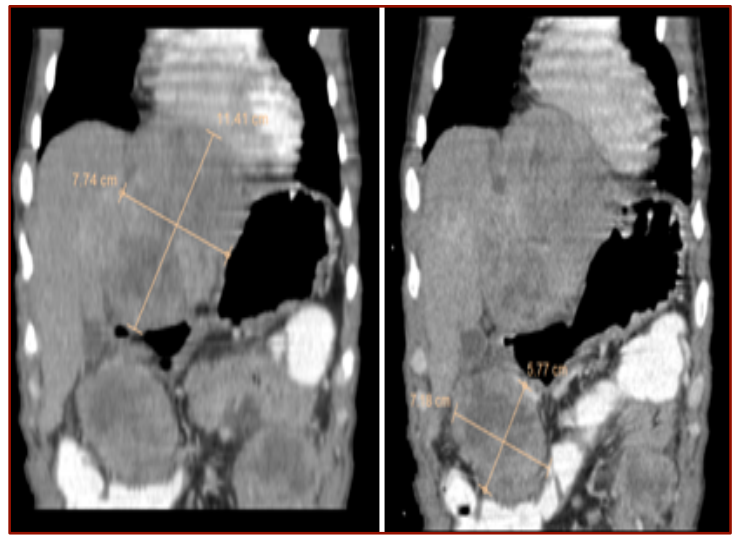

Figure Legend 2: (A, B) - Coronal section of multiple GISTS in the peritoneal cavity.

\section{DISCUSSION}

Gastrointestinal stromal tumors are most common subepithelial neoplasm that can be found throughout the gastrointestinal tract, most of them occur in stomach $\left({ }^{i}\right)$. GISTs can arise anywhere along the GIT; however, in the colon, stomach, and small intestine. GISTS are the most common mesenchymal tumors (2). Akwari et al. in their series, found that the largest numbers of tumors were from the stomach, followed by the small intestine. Tumors may also arise from the duodenum, large bowel, and even the peritoneum (4). Because most of these tumors are submucosal in location, these usually attain a large size without causing bowel obstruction by the time of diagnosis (2). Most of these tumors have an exophytic component as they arise from the muscularis propria. The enhancement pattern can vary from homogenously enhancing to heterogeneously enhancing, with or without ulceration, large heterogeneously enhancing masses due to areas of necrosis or cystic degeneration. Large size, hepatic metastasis and presence of wall invasion often suggest a high-grade GIST and predict poor outcome. Malignant GIST commonly metastasizes to the liver or peritoneum, whereas metastases to the lymph nodes and extra-abdominal metastases are rare (3). The differential diagnosis for small intestinal GISTs comprises of adenocarcinoma and lymphoma. Adenocarcinoma typically exhibits as an annular lesion in the proximal small intestine; thus, its appearance does not overlap with that of GISTs. Lymphoma, however, has many features similar to those of GISTs. The presence of related lymphadenopathy, however, would favor the diagnosis of lymphoma. The CT characteristics of metastatic lesions of GISTs are alike to those of primary tumors: enhancing masses that can be heterogeneous because of necrosis, hemorrhage, or cystic degeneration (1). 


\section{ABBREVIATIONS}

GISTs: gastro intestinal stromal tu

\section{REFERENCES}

1. Chourmouzi Danai, Sinakos Emmanouil et al. Gastrointestinal Stromal Tumors: a Pictorial Review. J Gastrointestin Liver Dis. J Gastrointestin Liver Dis. 2009 Sep;18(3):379-83.

2. Sripathi Smiti, Rajagopal KV, Srivastava Kumar Rajendra. CT features, mimics and atypical presentations of gastrointestinal stromal tumor (GIST). Indian journal of radiology and imaging. 2011;21(3) : 176-181.

3. Gong Jingshan, Kang Wenyan, Zhu,Jin et al. CT and MR imaging of gastrointestinal stromal tumor of stomach: a pictorial review. Quant Imaging Med Surg. 2012 Dec; 2(4): 274-279.

4. Akwari OE, Dozois RR, Weiland LH, Beahrs OH. Leiomyosarcoma of the small and large bowel. Cancer $1978 ; 42: 1375-84$

How to cite this article -Lakhar B, Guru N, Malignant Gastrointestinal Stromal Tumour with Metastasis, IJRSMS, 2015;01(1): 31 - 33 